\title{
Aflatoxin Biosynthesis and Genes of Aspergillus parasiticus
}

\author{
Kimiko Yabe
}

\begin{abstract}
Aflatoxins are produced from acetyl CoA through more than 18 enzyme steps. Although most of all enzymatic reactions have already been reproduced by in vivo and in vitro experiments, a few enzyme activities such as one catalyzing reaction from $O$ methylsterigmatocystin to aflatoxin $\mathrm{G}_{1}$ remained to be clarified.

We recently detected biosynthetic activities for aflatoxins $G_{1}$ and $G_{2}$ in cell-free extracts of $A$. parasiticus NIAH-26. In the presence of NADPH, aflatoxins $\mathrm{G}_{1}$ and $\mathrm{G}_{2}$ were produced from $O$-methylsterigmatocystin and dihydro- $O$-methylsterigmatocystin, respectively. Also, we for the first time determined that $\mathrm{G}$ - and B-aflatoxins are independently produced from the same substrate. At least three reactions, catalyzed respectively by the $\operatorname{ordA}$ gene product, an unstable microsome enzyme, and an $220-\mathrm{kDa}$ cytosol protein, are involved in the enzymatic formation of $\mathrm{G}$-aflatoxins from either $O$-methylsterigmatocysin or dihydro- $O$ methylsterigmatocystin.

On the other hand, many of the genes involved in aflatoxin biosynthesis have been isolated from $A$. parasiticus and $A$. flavus, and most of them have been found to be clustered. We recently purified $O$-methyltransferase catalyzing the conversion of demethylsterigmatocystin to sterigmatocystin in aflatoxin biosynthesis. Based on the $\mathrm{N}$-terminal amino acid sequence of the enzyme, genomic and cDNA genes $(d m t A)$ of the enzyme were then cloned and sequenced from $A$. parasiticus NIAH-26 through the use of polymerase chain reaction (PCR) strategies. The $d m t A$ gene was found to be placed between the $o m t A$ and ord-2 genes in the aflatoxin biosynthesis cluster region with the same orientation. Clarification of the aflatoxin biosynthetic mechanism using the biochemical and molecular biological approaches will provide promising clues to eliminate aflatoxin contamination from crops.
\end{abstract}

Key words: Aspergillus parasiticus, aflatoxin, biosynthesis

\section{Introduction}

Aflatoxins $\mathrm{B}_{1}\left(\mathrm{AFB}_{1}\right), \mathrm{B}_{2}\left(\mathrm{AFB}_{2}\right), \mathrm{G}_{1}\left(\mathrm{AFG}_{1}\right)$, and $\mathrm{G}_{2}\left(\mathrm{AFG}_{2}\right)$ are toxic, carcinogenic secondary metabolites produced by some strains of Aspergillus flavus, A. parasiticus, $A$. nomius, and $A$. tamarii. Contamination of feed and food with aflatoxins can have serious effect on health of animals and human. Many researchers have studied aflatoxin biosynthetic mechanism for the purpose of development of methods for preventing aflatoxin contami-

National Food Research Institute, Tsukuba, Ibaraki 305-8642, Japan

Tel : 0298-38-8050, Fax : 0298-38-7996 
nation.

All aflatoxins are produced form acetyl CoA through complicated pathways consisting of more than 18 enzyme steps ${ }^{1}$. For the determination of the pathways, two kinds of methods, in vivo feeding experiment and in vitro enzyme assay, have been mainly used. Although most of all reaction steps had already been determined by the both in vivo and in vitro experiments, a few enzyme activities such as ones catalyzing the convesion of acetyl CoA to hexanoyl CoA, hexanoyl CoA to norsolorinic acid, averufin to versiconal hemiacetal acetate, versicolorin A to demethylsterigmatocystin, and $O$-methylsterigmatocystin (OMST) to $A F G_{1}$ remained to be clarified.

In this study, we investigated the enzyme activity for the formation of G-group aflatoxins in detail ${ }^{2}$. The biosynthetic pathway(s) associated with the formation of G-group aflatoxins has long been controversial. Some researchers have reported that in feeding experiments radioactive $\mathrm{AFB}_{1}$ was converted to G-group aflatoxins, while other have not observed this ${ }^{3}$. However, it is generally assumed that G-group aflatoxins are produced from B-aflatoxins by insertion of oxygen into a $\mathrm{C}-\mathrm{C}$ bond through a Baeyer-Villigar reaction. We previously reported enzymatic conversion of OMST to $\mathrm{AFB}_{1}$ or dihydro- $O$ methylsterigmatocystin (DHOMST) to $\mathrm{AFB}_{2}$ by using cell-free extract of Aspergillus parasiticus NIAH-26 and thin layer chromatography analysis ${ }^{4}$. In the course of the similar experiments using OMST as a substrate, faint band corresponding to $\mathrm{AFG}_{1}$ together with clear bands of $\mathrm{AFB}_{1}$ were sometimes observed. Therefore, in order to confirm the production of G-group aflatoxins in more detail, we used HPLC analyses for the cell-free assays. Finally, we determined the enzyme activity for G-group aflatoxins.

On the other hand, many genes of the enzymes and the transcription factor involved in aflatoxin biosynthesis have been cloned and characterized, following determination of the biosynthetic pathways ${ }^{5}$. These genes have been shown to form a gene cluster located in an approximately $75-\mathrm{kb}$ region in the $A$. flavus and $A$. parasiticus genomes, although some differences between different species exist. Furthermore, many fungi such as $A$. nidulans and Bipolaris produce sterigmatocystin (ST), a precursor of aflatoxins, and it is suggested that almost the same pathways as that for aflatoxin biosynthesis may also be involved in sterigmatocystin biosynthesis; in fact, similar genes as those in the aflatoxin biosynthesis have been isolated from $A$. nidulans, and it has been found that these genes are also located in a $60-\mathrm{kb}$ cluster of the $A$. nidulans genome ${ }^{6}$. Determination of enzyme activity makes it possible to isolate the gene coding the enzyme. Thus, we determined the gene (named as $d m t A$ ) coding $O$-methyltransferase I (MT-I) catalyzing the conversion of demethylsterig matocystin to sterigmatocystin and of dihydrodemethylsterigmatocystin to dihydrosterigmatocystin $^{7}$.

\section{Materials and Methods}

Microorganisms and cultures $A$. parasiticus NIAH-26, a UV-irradiated mutant of $A$. parasiticus SYS-4 (NRRL 2999) was used ${ }^{8)}$. Fungal strains were cultured at static state at $28^{\circ}$ $\mathrm{C}$ for 4 days either in a YES liquid medium, an aflatoxin-inducing medium, or in a YEP liquid medium (2\% yeast extract, $20 \%$ peptone), an aflatoxin-non-inducing medium.

Enzyme assays for the aflatoxin formation Cell-free extract, microsome, and cytosol fractions were prepared from the mycelia of $A$. parasiticus NIAH-26 grown in YES medium 
at $28^{\circ} \mathrm{C}$ for 4 days by grinding the mycelia and then successive centrifugations ${ }^{9)}$. We devised assay conditions for optimizing the enzyme activity. The cell-free extracts $(1.3 \mathrm{mg}$ of protein per $\mathrm{ml}$ ) was incubated in a reaction mixture consisting of $90 \mathrm{mM}$ potassium phosphate [pH 7.5], $10 \%$ [vol/vol] glycerol supplemented with $80 \mu \mathrm{M}$ of each substrate, $4 \mathrm{mM}$ $\mathrm{NADPH}, \mathrm{BSA}(0.9 \mathrm{mg} / \mathrm{ml})$, and phosphatidyl choline liposome $(0.5 \mathrm{mg} / \mathrm{ml})$. The final volume of the reaction mixture was $50 \mu$. Reactions were routinely carried out at $24^{\circ} \mathrm{C}$ for 40 min and then terminated by adding water-saturated chloroform and mixing with a Vortex mixer. An aliquot of the lower chloroform layer was directly injected into a Shimadzu LC-6A HPLC equipped with a silica gel HPLC column. The solvent system consisted of toluene/ethyl acetate/formic acid/methanol (198:15:4:3, v/v/v/v). The fluorescence intensity of each aflatoxin was monitored with a fluorescence monitor (ex. was $365 \mathrm{~nm}$; em. was $425 \mathrm{~nm}$ ) with a flow rate of $1 \mathrm{ml} / \mathrm{min}$ at room temperature.

Determination of the amino acid sequence of the MT-I MT-I was purified as described previously ${ }^{10}$, and analyzed by sodium dodecyl sulfate-polyacrylamide gel electrophoreses using $13 \%$ polyacrylamide gel. The protein was blotted onto a polyvinylidine difluoride membrane (immobilon P), and the part corresponding to MT-I in the membrane was cut and then applied onto an automated Edman degradation gas phase sequencer.

cDNA synthesis and 5'- and 3'-RACE PCR To obtain the full-length cDNA sequence, mRNA ( $1 \mu \mathrm{g}$ ) was used to generate double-stranded cDNA using the Marathon cDNA Amplification kit (CLONTECH Laboratory, Inc., Palo Alto, CA), and the double-stranded cDNA was ligated with the Marathon cDNA adaptor. 3'-RACE and 5'-RACE were performed using the double-stranded $c D N A$ as a template with a $d m t A$ gene-specific primers and the adaptor primer AP1. The PCR products of both 5'-RACE and 3'-RACE were gelpurified, ligated into vector TA, and sequenced.

Cloning of genomic DNA and TAIL-PCR DNA was purified from $A$. parasiticus NIAH-26. To determine the internal sequence of the genomic $d m t A$ gene, PCR reactions were carried out using the genomic DNA of $A$. parasiticus NIAH-26 as a template with various primers having the cDNA sequence, and the resultant PCR products were sequenced. To determine the sequences of the 5'- as well as the 3'-flanking regions, thermal asymmetric interlaced (TAIL) PCR ${ }^{10)}$ was used.

Northern analysis The spore suspension was inoculated into $100 \mathrm{ml}$ of either SY liquid medium (6\% sucrose, $2 \%$ yeast extract) as an aflatoxin-inducing medium or PY liquid medium ( $4 \%$ peptone and $2 \%$ yeast extract) as an aflatoxin-non-inducing medium, and incubated at either $28^{\circ} \mathrm{C}$, permissive temperature of aflatoxin production, or at $37^{\circ} \mathrm{C}$, nonpermissive temperature, for 3 days on a rotary shaker, and then the mycelia were harvested. The membranes the total RNA was blotted onto were then probed with the DIG-labeled DNA fragment, and the hybridized DNA band was immunodetected by using the Detection DIG Luminescent Detection Kit (Boehringer Mannheim).

DNA sequence analysis. All PCR products were ligated into TA-cloning vector, pCR 2.1, and then transformed into bacteria, INVaF (Invitrogen BV, The Netherlands), and sequenced.The genomic and cDNA nucleotide sequence data of $d m t A$ are available from DDBJ/ 
EMBL/GenBank nucleotide sequence databases with accession numbers AB022905 and AB022906.

\section{Results}

Detection of enzyme activity for G-group aflatoxin formation When the cell-free extract of $A$. parasiticus NIAH-26 was incubated with OMST in the presence of NADPH at $24^{\circ} \mathrm{C}$ for $40 \mathrm{~min}, \mathrm{AFG}_{1}$ as well as $\mathrm{AFB}_{1}$ were formed. When DHOMST, instead of OMST, was added to the reaction mixture, $\mathrm{AFG}_{2}$ and $\mathrm{AFB}_{2}$ were produced. The amount of $\mathrm{AFG}_{1}$ produced from OMST continued to increase for at least $40 \mathrm{~min}$. We found that co-presence of lipid $(0.5$ $\mathrm{mg} / \mathrm{ml})$ and $\mathrm{BSA}(0.9 \mathrm{mg} / \mathrm{ml})$ in the reaction mixture maximized the enzyme activity. The formation of $A F G_{1}$ and $A F B_{1}$ was reduced by $3 \mathrm{mM}$ of methyrapone, SKF-525A, or imidazole, indicating that the formation of both $A F B_{1}$ and $A F G_{1}$ were dependent upon cytochrome P-450 oxygenase activity.

The enzyme activity for G-AF formation using various metabolites as substrates were measured. $\mathrm{AFG}_{1}$ was produced from $\mathrm{OMST}$, and $\mathrm{AFG}_{2}$ was from DHOMST. However, neither $\mathrm{AFG}_{1}$ nor $\mathrm{AFG}_{2}$ was produced from either $\mathrm{AFB}_{1}$ or $\mathrm{AFB}_{2}$, nor did the reverse reaction from G-group aflatoxins to B-group aflatoxins occurred. These results indicate that $\mathrm{B}$ group aflatoxins are not the precursors of G-group aflatoxins, and that G- and B-group aflatoxins are independently produced from the same substrate (OMST for $A F G_{1}$ and $A F B_{1}$, and $\mathrm{DHOMST}$ for $\mathrm{AFG}_{2}$ and $\mathrm{AFB}_{2}$ ) through different pathways from a common branching intermediate.

To determine the relationship between $\mathrm{AFG}_{1}$ and $\mathrm{AFG}_{2}$ formation, we examined the enzyme activities by using reaction mixtures that contained $5 \mu \mathrm{M}$ DHOMST and different concentrations of OMST. Although $\mathrm{AFG}_{2}$ was produced from DHOMST, the amount formed decreased with increasing OMST concentration and $\mathrm{AFG}_{1}$ newly formed. Addition of high concentrations of OMST (more than $10 \mu \mathrm{M}$ ) led to exclusive formation of $\mathrm{AFG}_{1}$. These results indicate that the same enzyme(s) may catalyze the formation of $A F G_{1}$ and $\mathrm{AFG}_{2}$.

Requirement of both the microsome fraction and 220-kDa protein for G-aflatoxin production We measured the G-aflatoxin formation activity by using either microsome or cytosol fractions or a combination of both. No significant amounts of $A F G_{1}$ were formed in the OMST + cytosol reaction, and the OMST+microsome reaction produced only trace of $\mathrm{AFG}_{1}$. When both the cytosol and microsome fractions were present, enzyme activity was markedly enhanced and dependent upon microsome concentration. We examined the activation factor in the cytosol fraction by fractionating it through an Ultrogel AcA 34 gel filtration column and then incubating each fraction with OMST in a reaction mixture containing the microsome fraction. $\mathrm{AFG}_{1}$ formation activity was enhanced threefold by the addition of the fraction that corresponded to a molecular weight of about $220 \mathrm{kDa}$. These results indicate that at least two kinds of enzymes, one a microsome enzyme and the other a $220-\mathrm{kDa}$ soluble protein, are necessary for the formation of $\mathrm{AFG}_{1}$ from OMST.

When the cell-free extract was incubated with or without liposome and BSA at $24^{\circ} \mathrm{C}$ for various times, $A F G_{1}$ production decreased to less than $50 \%$ of the original activity level after $15 \mathrm{~min}$ and to less than $20 \%$ after $30 \mathrm{~min}$, whereas the $\mathrm{AFB}_{1}$ formation activity was more stable than was that for AF. It was found that the microsome enzyme is the cause of 
instability observed for G-group aflatoxin biosynthesis.

Cloning and nucleotide sequencing of MT-I cDNA and its deduced amino acid sequence We purified MT-I from $A$. parasiticus NIAH-26 to homogeneity. Its molecular weight was $43 \mathrm{kDa}$ (SDS-PAGE) and the $N$-terminal sequence was: T-G-L-D-M-E-I-I-F-A-K-I-K-E-EY-A-R-T-D-D-V-G-K-R-Q-I-Q-G-H-I-R-E-L-Q-V-G-F-Y-S(P)-D-L(W)-D-V-V-M-R-L-A(S)-SG-. In order to determine the specific nucleotide sequence of $d m t A$, we used degenerate oligonucleotides corresponding to the $N$-terminal sequence of the MT-I protein. A 144-bp PCR product was obtained.

We then used 5'-RACE and 3'-RACE techniques to determine the cDNA sequence of $d m t A$ gene. Conventional and TAIL-PCR were further used to determine the genomic DNA sequence $^{7}$. The overall cDNA sequence was determined to be $1373 \mathrm{bp}$, and first $\mathrm{M}$ may later be processed post-translationally. The open reading frame of the cDNA codes for a 386-amino-acid-residue protein, and the theoretical molecular mass without the $N$-terminal methionine was calculated to be $43,023 \mathrm{Da}$, which corresponded well with the mass determined by SDS-PAGE $(43 \mathrm{kDa})^{10}$. DmtA (=MT-I) contains a consensus motif I of $S$-adenosylmethionine-dependent methyltransferase in the central region.

By comparison of the cDNA sequence with the genomic DNA sequence, the coding sequence (nucleotide 1/1333) of $d m t A$ was found to be separated with three introns. In addition, the palindrome sequence TCG(N5)CGA, which has been suggested to be a possible binding region of AflR in $A$. nidulans ${ }^{12)}$, was found. Three TATAA sequences close to the transcription initiation site were also found.

Using TAIL-PCR, it was found that the sequence of the region of $-703 /-499$ overlapped with the 3 '-flanking region of the omtA gene. We also found that the 3 '-flanking region $(1415 / 1683)$ of $d m t A$ overlapped with the 5'-cDNA sequence of ord-2. The orientations of $d m t A$ and $o r d-2$ were also the same.

Expression of the $\boldsymbol{d m \boldsymbol { t } A}$ gene Transcription of $d m t A$ gene was determined by Northern blotting. When $A$. parasiticus NIAH-26 was cultured at $28^{\circ} \mathrm{C}$ for 3 days in an aflatoxininducing SY medium, a 1.4 -kb transcript of $d m t A$ was seen. In contrast, when an aflatoxinnon-inducing PY medium was used instead of SY, $d m t A$ was not expressed. Also, when the fungi were cultured at a higher temperature $\left(37^{\circ} \mathrm{C}\right)$, an aflatoxin-non-permissive temperature ${ }^{13)}$, the expression of $d m t A$ was not detected, irrespective of the kind of medium.

\section{Discussion}

We detected enzyme activity for $\mathrm{G}$-aflatoxin formation by using a cell-free extract or a combination of cytosol and microsome fractions of $A$. parasiticus NIAH-26. The relative week activity of G-aflatoxin biosynthesis may indicate that the enzymes for G-aflatoxin formation are relatively sensitive to the preparation of the cell-free fractions, whereas the enzymes for B-aflatoxin formation are much less sensitive to this process. Furthermore, $\mathrm{G}$-aflatoxin biosynthesis requires both cytosol and microsome fractions and, if only one of these fractions was used for examining G-aflatoxin biosynthesis, then no G-aflatoxin would have been detected. These findings may at least partly explain why the enzyme activity for G-aflatoxin biosynthesis had not been previously detected.

In this study, we for the first time determined independent formation of G- and B-group 


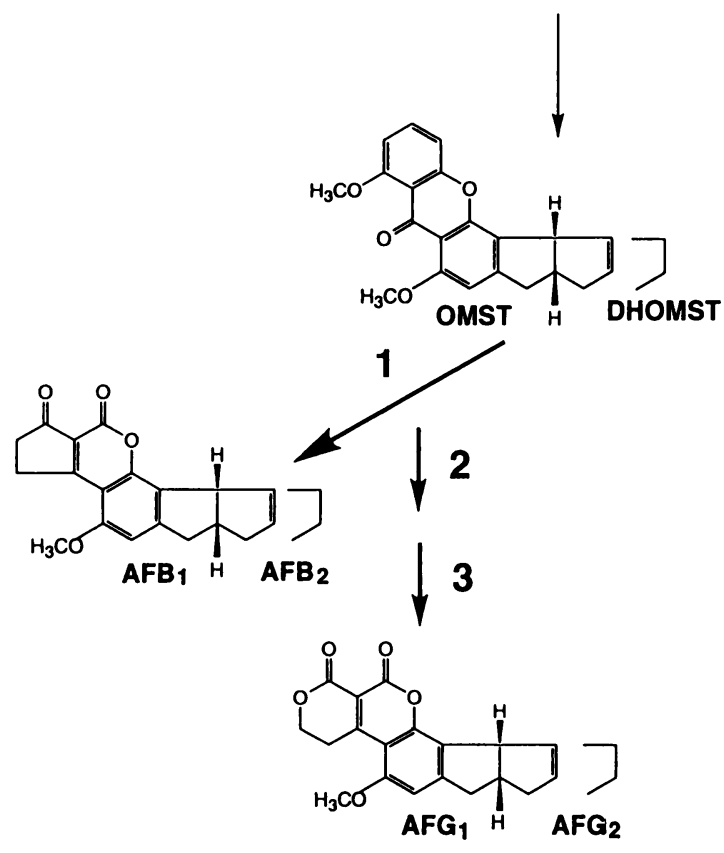

Fig. 1 Metabolic pathway for formation of $\mathrm{AFG}_{1}$ from $\mathrm{OMST}$ and formation of $\mathrm{AFG}_{2}$ from DHOMST.

At least three enzyme reactions may be commonly included in both formations. Reaction 1 may be catalyzed by an ordA gene product, which is microsome cytochrome P-450 oxygenase. A certain transient intermediate formed during this pathway may be subsequently converted to another substance in reaction 2 , and finally converted to the final product, $\mathrm{AFG}_{1}$ or $\mathrm{AFG}_{2}$ in reaction 3. Reactions 2 and 3 may be catalyzed by an unstable microsome enzyme and a $220-\mathrm{kDa}$ cytosol protein. However, the order of these three enzymes was not determined in this study.

aflatoxins. $\mathrm{AFG}_{1}$ and $\mathrm{AFG}_{2}$ were produced from OMST and DHOMST, respectively, whereas neither $\mathrm{AFG}_{1}$ nor $\mathrm{AFG}_{2}$ was produced from $\mathrm{AFB}_{1}$ or $\mathrm{AFB}_{2}$ in the cell-free systems. Recently, ord1 gene and ordA gene were isolated from $A$. flavus ${ }^{14)}$ and from $A$. parasiticus ${ }^{15)}$, respectively, and were confirmed to be required for the conversion of OMST to $\mathrm{AFB}_{1}$. Yu et al. ${ }^{15)}$ reported that complementation of $A$. parasiticus SRRC 2043, an OMST-accumulating strain, with the $\operatorname{ord} A$ gene restores the ability to produce $A F B_{1}, A F B_{2}, A F G_{1}$, and $A F G_{2}$, indicating that $\operatorname{ordA}$ gene product may be needed for the formation of $\mathrm{G}$-group aflatoxins.

However, our results show differences between the biosynthetic activities for G-AF and $\mathrm{B}-\mathrm{AF}$, because the microsome enzyme fraction involved in $\mathrm{G}$-formation was less stable than that for B-formation. Therefore, at least three enzymes appeared to be required for conversion of OMST to $\mathrm{AFG}_{1}$ and that of DHOMST to $\mathrm{AFG}_{2}$ (Fig. 1): a microsome ordA gene product, an unstable microsome protein, and a $220 \mathrm{kDa}$ cytosol protein. Although $\mathrm{B}$ aflatoxins may be the final products of the reactions catalyzed by the ordA product, more than two reactions may be included during the pathway either from OMST to $\mathrm{AFB}_{1}$ or from DHOMST to $\mathrm{AFB}_{2}$.

This study gives a final conclusion to long controversy about $\mathrm{G}$-aflatoxin biosynthesis. It is generally accepted that stains of $A$. parasiticus produce both $\mathrm{G}$ - and $\mathrm{B}$-aflatoxins, while 
A. flavus strains produce only the B-aflatoxins. Also, A. nidulans dose not make aflatoxins, because sterigmatocystin and dihydrosterigmatocystin are their final products. This study is showing a clue to elucidate the differences in aflatoxin productivity among the fungi.

On the other hand in the current study, we determined the cDNA and genomic DNA sequences encoding $O$-methyltransferase I from $A$. parasiticus. This gene is present as a single-copy in the genome, and is placed between the omt $A$ and ord-2 genes within the aflatoxin biosynthesis-gene cluster.

Kelkar $e t a l^{16)}$. have found by gene disruption strategy that an open reading frame from 42597 to 41970 (ENU 34740) of the $A$. nidulans stcP gene encodes MT-I. Since the $d m t A$ gene of $A$. parasiticus is supposed to be a homolog of the stcP gene, the deduced amino acid sequences of both genes were compared. The $d m t A$ gene product showed a $74.4 \%$ identity and $87 \%$ similarity to the deduced gene product encoded by a nucleotide sequence of the 1289-bp region (43273-41985), which covers the region containing 5 '-flanking region of the $s t c P$ gene and the gene itself. This $s t c P$ whole region may code a protein composed of 424 amino acids, and its theoretical molecular mass is $47,840 \mathrm{Da}$.

Gene walking using TAIL-PCR technology revealed that the $d m t A$ gene is placed between the $o m t A$ gene and the ord-2 genes in the same direction. The region between $o m t A$ and $d m t A$ contains the TATA sites and the AffR recognition site ${ }^{12)}$, indicating that the expression of $d m t A$ is also regulated by the affR gene product. This was also confirmed by the results showing that the expression of $d m t A$ has the same dependency on the culture temperature and the culture medium.

At least a part of the aflatoxin biosynthetic pathways has commonly been detected in many species. Although $A$. flavus and $A$. parasiticus can be expected to have whole aflatoxin gene cluster leading to the formation of aflatoxins, other fungi such as $A$. nidulans contain genes until sterigmatocystin formation and so on. The each coding region of each gene are similar among the different fungi, whereas the distribution of each genes and the non-coding regions are remarkably different. Evolutional relationship among the genes seems to be interesting. Based on these knowledge, it will be possible that the useful methods for prevention of the aflatoxin contamination will be obtained in the near future.

\section{Acknowledgment}

These works have been carried out by corroboration with Prof. Hamasaki's group, Faculty of Agriculture, Tottori University, and Ms. Marisa Motomura and Prof. Takao Shinozawa, Faculty of Engineering, Gunma University, Japan. We thank Dr. K. Yamagishi, Tohoku National Agricultural Experiment Station, Japan, for supplying all random primers for TAIL-PCR.

\section{References}

1) Yabe, K. (1998) Biosynthetic pathways of aflatoxins. Nippon Nogeikagaku Kaishi. 72:63-67 (in Japanese).

2) Yabe, K., Nakamura, M., Hamasaki, T. (1999) Enzymatic formation of G-group aflatoxins and biosynthetic relationship between G- and B-group aflatoxins. Appl. Environ. Microbiol. 65 : 38673872 .

3) Henderberg, A., Bennett, J. W., L. S. Lee.(1988) Biosynthetic origin of aflatoxin $\mathrm{G}_{1}$ : confirmation 
of sterigmatocystin and lack of confirmation of aflatoxin $B_{1}$ as precursors. J. Gen. Microbiol. 134: $661-667$

4) Yabe, K., Ando, Y., Hamasaki, T. 1988. Biosynthetic relationship among aflatoxins $B_{1}, B_{2}, G_{1}$, and $\mathrm{G}_{2}$. Appl. Environ. Microbiol. 54 : 2101-2106.

5) Woloshuk, C. P., Prieto, R. 1998. Genetic organization and function of the aflatoxin $B_{1}$ biosynthetic genes. FEMS Microbiology Letters $160: 169-176$.

6) Brown, D. W., Yu, J.-H., Kelkar, H. S., Fernandes, M., Nesbitt, T. C., Keller, N. P., Adams, T. H., Leonard, T. J. (1996) Twenty-five coregulated transcripts define a sterigmatocystin gene cluster in Aspergillus nidulans. Proc. Natl. Acad. Sci. USA 93: 1418-1422.

7) Motomura, M., Chihaya, N., Shinozawa, T., Hamasaki, T., Yabe, K. (1999) Cloning and characterization of the $O$-methyltransferase I gene $(d m t A)$ from Aspergillus parasiticus associated with the conversions of demethylsterigmatocystin to sterigmatocystin and dihydrodemethylsterigmatocystin to dihydrosterigmatocystin in aflatoxin biosynthesis. Appl. Environ. Microbiol. 65 : 4987-4994.

8) Yabe, K., Nakamura, H., Ando, Y., Terakado, N., Nakajima, H., Hamasaki, T. (1988) Isolation and characterization of Aspergillus parasiticus mutants with impaired aflatoxin production by a novel tip culture method. Appl. Environ. Microbiol. 54 : 2096-2100.

9) Yabe, K., Ando, Y., Hamasaki, T. (1991) A metabolic grid among versiconal hemiacetal acetate, versiconol acetate, versiconol and versiconal during aflatoxin biosynthesis. J. Gen. Microbiol. 137 : 2469-2475.

10) Yabe, K., Matsushima, K.-I., Koyama, T., Hamasaki, T. (1998) Purification and characterization of $O$-methyltransferase I involved in the conversion of demethylsterigmatocystin to sterigmatocystin and of dihydrodemethylsterigmatocystin to dihydrosterigmatocystin during aflatoxin biosynthesis. Appl. Environ. Microbiol. 64:166-171.

11) Liu, Y.-G., Whittier, R. F. (1995) Thermal asymmetric interlaced PCR: automatable amplification and sequencing of insert and fragments from P1 and YAC clones for chromosome walking. Genomics $25: 674-681$

12) Fernandes, M., Keller, N. P., Adams, T. H. (1998) Sequence-specific binding by Aspergillus nidulans AflR, a C6 zinc cluster protein regulating mycotoxin biosynthesis. Mol. Microbiol. 28: 1355-1365.

13) Feng, G. H., Leonard, T. J. (1998) Culture conditions control expression of the genes for aflatoxins and sterigmatocystin biosynthesis in Aspergillus parasiticus and A. nidulans. Appl. Environ. Microbiol. 64 : 2275-2277.

14) Prieto, R., Woloshuk (1997) ord1, an oxidoreductase gene responsible for the conversion of $O$-methylsterigmatocystin to aflatoxin in Aspergillus flavus. Appl. Environ. Microbiol. 63: 1661-1666.

15) Yu, J., Chang, P.-K., Payne, G. A., Cary, J. W., Bhatragar, D. Cleveland T. E. (1995) Comparison of the omt $A$ genes encoding $O$-methyltransferases involved in aflatoxin biosynthesis from Aspergillus parasiticus and $A$. flavus. Gene $163: 121-125$.

16) Kelkar, H. S., Keller, N. P., Adams, T. H. (1996) Aspergillus nidulans stcP encodes an $O$ methyltransferase that is required for sterigmatocystin biosynthesis. App. Environ. Microbiol. 62 : 4296-4298. 